\title{
On the relation between low-energy constants and resonance saturation
}

\author{
Maarten Golterman* and Santiago Peris \\ Grup de Física Teòrica and IFAE \\ Universitat Autònoma de Barcelona, 08193 Barcelona, Spain
}

\begin{abstract}
Although there are phenomenological indications that the low-energy constants in the chiral lagrangian may be understood in terms of a finite number of hadronic resonances, it remains unclear how this follows from QCD. One of the arguments usually given is that low-energy constants are associated with chiral symmetry breaking, while QCD perturbation theory suggests that at high energy chiral symmetry is unbroken, so that only low-lying resonances contribute to the low-energy constants. We revisit this argument in the limit of large $N_{c}$, discussing its validity in particular for the low-energy constant $L_{8}$, and conclude that QCD may be more subtle that what this argument suggests. We illustrate our considerations in a simple Regge-like model which also applies at finite $N_{c}$.
\end{abstract}

*Permanent address: Department of Physics and Astronomy, San Francisco State University, 1600 Holloway Ave, San Francisco, CA 94132, USA 


\section{Introduction}

The low-energy physics of the Goldstone bosons associated with the spontaneous breaking of chiral symmetry is described by the chiral lagrangian, the construction of which is solely based on the general principles of quantum field theory and the symmetries of the underlying fundamental theory of the strong interactions, QCD. The only "dynamical" information present in the chiral lagrangian connecting it to QCD is encompassed by the values of the low-energy constants (LECs) that parameterize the terms appearing in the chiral lagrangian. At lowest order, there are two LECs, the pion decay constant $f_{\pi}$ and the quark condensate $\langle\bar{\psi} \psi\rangle$, whereas at higher orders, a growing number of LECs appear. In the theory with three flavors, there are ten physical LECs at order $p^{4}$ [1], denoted as $L_{i}, i=1, \ldots, 10$.

It is thus an interesting question to ask what physics of the strong interaction leads to the values of the LECs observed in nature. Since the physical spectrum contains infinite towers of resonances with given quantum numbers (spin, flavor and parity), it is natural to think of the values of the order $p^{4}$ (and higher-order) LECs as resulting from integrating out these resonances $\left[2,3 .{ }^{1}\right.$ In particular, sum rules have been derived relating the order- $p^{4}$ constants to the masses and decay constants of these resonances.

Here we revisit in particular the sum rule relating $L_{8}$ to the scalar and pseudoscalar resonances with isospin 1 , working in the large- $N_{c}$ and chiral limits. The choice of taking $N_{c}$ large simplifies the discussion because hadronic resonances are stable in that limit. Furthermore, it is natural to work in the chiral limit, since the LECs are independent of the quark masses. For comparison, we will also discuss the sum rule relating $L_{10}$ to vector and axial-vector resonances with isospin 1.

We begin with reviewing the usual type of sum rule relating $L_{8}$ to resonance parameters through the difference of scalar and pseudoscalar two-point functions $\Pi_{S}\left(q^{2}\right)-$ $\Pi_{P}\left(q^{2}\right)$, and show that it is in principle ill-defined. We show that the problem originates from the fact that each of these two-point functions is quadratically divergent in QCD. We will then derive a new sum rule, starting from a subtracted dispersion relation, in Sec. 2.2. In Sec. 3 we give an example of how the new sum rule works, using a simple Regge-like model in which the exact answer is known. This model allows us also to consider the situation at finite $N_{c}$. In Sec. 4 we test the phenomenological approach in which the difference $\Pi_{S}\left(q^{2}\right)-\Pi_{P}\left(q^{2}\right)$ is saturated by a finite number of resonances. The usual argument for this approach is that this difference vanishes in perturbation theory, suggesting that the contribution from resonances higher in the spectrum cancels, at least approximately. Applying this approach to our model, we find that the effective parameters (i.e. masses and coupling constants) which are required to fit high-energy constraints as well as to yield the correct value for $L_{8}$ are not the same as those of the original infinite spectrum. The final section contains our conclusions.

\footnotetext{
${ }^{1}$ The physics of $f_{\pi}$ and $\langle\bar{\psi} \psi\rangle$ is associated with the breaking of chiral symmetry by the vacuum.
} 


\section{The large- $N_{c}$ limit}

\subsection{Review}

In the large- $N_{c}$ limit, $L_{8}$ is given by [4]

$$
16 B^{2} L_{8}=\Delta \Pi(0)=\frac{1}{2}\left(\Pi_{S}(0)-\Pi_{P}^{\prime}(0)\right),
$$

in which the chiral limit is taken, and where we define

$$
\Pi_{S, P}\left(q^{2}\right)=i \int d^{4} x e^{i q x}\left\langle 0\left|T\left\{J_{S, P}(x) J_{S, P}^{\dagger}(0)\right\}\right| 0\right\rangle,
$$

with $J_{S}(x)=\bar{d}(x) u(x)$ and $J_{P}(x)=\bar{d}(x) i \gamma_{5} u(x)$. The order- $p^{2}$ constant $B$ is equal to

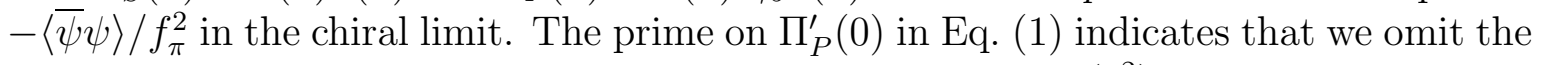
pion pole present in the pseudoscalar two-point function $\Pi_{P}\left(q^{2}\right)$. Since the difference $\Pi_{S}-\Pi_{P}$ cancels in perturbation theory for vanishing quark masses, this means that $\Delta \Pi\left(q^{2}\right)$ obeys an unsubtracted dispersion relation in the chiral limit, i.e.

$$
\Delta \Pi\left(q^{2}\right) \equiv \frac{1}{2}\left(\Pi_{S}\left(q^{2}\right)-\Pi_{P}^{\prime}\left(q^{2}\right)\right)=\frac{1}{2 \pi} \int_{0}^{\infty} d t \frac{\operatorname{Im}\left(\Pi_{S}(t)-\Pi_{P}^{\prime}(t)\right)}{t-q^{2}-i \epsilon} .
$$

Equation (II) states, then, that $L_{8}$ becomes a physical quantity at $N_{c}=\infty$. In this limit, there is an infinite tower of infinitely narrow resonances in each channel, and we thus have that [5]

$$
\begin{aligned}
& \frac{1}{\pi} \operatorname{Im} \Pi_{S}(t)=2 \sum_{n}^{\infty} F_{S}^{2}(n) \delta\left(t-M_{S}^{2}(n)\right), \\
& \frac{1}{\pi} \operatorname{Im} \Pi_{P}^{\prime}(t)=2 \sum_{n}^{\infty} F_{P}^{2}(n) \delta\left(t-M_{P}^{2}(n)\right) .
\end{aligned}
$$

Use of Eq. (4) in Eq. (3) leads formally to

$$
\Delta \Pi\left(q^{2}\right)=\sum_{n}^{\infty} \frac{F_{S}^{2}(n)}{M_{S}^{2}(n)-q^{2}-i \epsilon}-\sum_{n}^{\infty} \frac{F_{P}^{2}(n)}{M_{P}^{2}(n)-q^{2}-i \epsilon} .
$$

In arriving at this expression for $\Delta \Pi\left(q^{2}\right)$, we assumed that the integral over $t$ and the sum over $n$ may be freely interchanged. Ignoring the question of validity of this assumption for the time being, one thus arrives at a sum rule for $L_{8}$ :

$$
16 B^{2} L_{8}=\sum_{n}^{\infty} \frac{F_{S}^{2}(n)}{M_{S}^{2}(n)}-\sum_{n}^{\infty} \frac{F_{P}^{2}(n)}{M_{P}^{2}(n)} .
$$

As it stands, this sum rule is not well-defined, because both sums appearing on the right-hand side are quadratically divergent. First, let us give an example. If we assume 
Regge-like behavior asymptotically for large $n$, with ${ }^{2}$

$$
\begin{aligned}
M_{S, P}^{2}(n) & \sim n \Lambda^{2}, \quad n \text { large }, \\
F_{S, P}^{2}(n) & \sim \kappa \Lambda^{2} M_{S, P}^{2}(n),
\end{aligned}
$$

with $\Lambda$ a constant of order $1 \mathrm{GeV}$, and $\kappa$ is equal to the coefficient of the parton model logarithm, then Eq. (6) looks like

$$
16 B^{2} L_{8} \sim \kappa \Lambda^{2}\left(\sum_{n}^{\infty} 1-\sum_{n}^{\infty} 1\right),
$$

which is clearly ill-defined. However, as we will now discuss, the problem is model independent. We will return to the model (7) in Sec. 3.

In order to proceed in the general case, let us introduce cutoffs $N_{S, P}$ on the summation index $n$ in the scalar and pseudoscalar channels, corresponding to cutoffs $M_{S}\left(N_{S}\right), M_{P}\left(N_{P}\right)$ on the masses, in order to regulate the sums. Furthermore, we require that both two-point functions match on to QCD perturbation theory for asymptotically large euclidean $Q^{2}=-q^{2}$. In particular, the leading large- $Q^{2}$ behavior is given by $\kappa Q^{2} \log Q^{2}$, with $\kappa$ a constant common to both channels, because the perturbative expressions for scalar and pseudoscalar two-point functions, valid for asymptotically large $Q^{2}$, are equal. It follows that indeed the number of resonances in each channel has to be infinite [5]. Furthermore, writing

$$
F_{S, P}^{2}(n)=f_{S, P}^{2}(n) M_{S, P}^{2}(n),
$$

each of the sums in Eq. (5) can be written as (omitting the indices $S$ or $P$ )

$$
\begin{aligned}
& \sum_{n}^{N} \frac{F^{2}(n)}{M^{2}(n)+Q^{2}}=\sum_{n}^{N} f^{2}(n)-Q^{2} \sum_{n}^{N} \frac{f^{2}(n)}{M^{2}(n)+Q^{2}} \\
& \sim \int^{M^{2}(N)} d M^{2}\left(\frac{d n}{d M^{2}} f^{2}(n)\right)-Q^{2} \int^{M^{2}(N)} d M^{2}\left(\frac{d n}{d M^{2}} f^{2}(n)\right) \frac{1}{M^{2}+Q^{2}} .
\end{aligned}
$$

If $\left(d n / d M^{2}\right) f^{2}(n)$ goes like a constant for large $M^{2}$, the second integral will behave like $\log \left(M^{2}(N) / Q^{2}\right)$ for $M^{2}(N) \gg Q^{2}$, thus yielding the desired parton-model logarithm. As a consequence, the first integral in Eq. (10) is quadratically divergent. Note that $M^{2}(N) \gg Q^{2}$ should hold for any $Q^{2}$, and that we thus need to require that $M^{2}(N) \rightarrow$ $\infty$ when $N \rightarrow \infty$. It follows that the constant $\kappa$ can be expressed as

$$
\kappa \equiv \lim _{n \rightarrow \infty} \frac{d n}{d M_{S}^{2}} f_{S}^{2}(n)=\lim _{n \rightarrow \infty} \frac{d n}{d M_{P}^{2}} f_{P}^{2}(n) .
$$

Furthermore, it also follows that $M_{S}^{2}\left(N_{S}\right) / M_{P}^{2}\left(N_{P}\right) \rightarrow 1$, to avoid a term linear in $Q^{2}$ in $\Pi_{S}\left(q^{2}\right)-\Pi_{P}\left(q^{2}\right)$, for $q^{2}=-Q^{2}$.

\footnotetext{
${ }^{2}$ For the asymptotic proportionality between decay constants and the masses, see Eq. (11) below.
} 
In fact, we may choose to introduce a common cutoff $\Lambda_{c o}^{2}$ on the $t$ integrals in Eq. (5), which implies cutoffs $N_{S, P}$ on the sums over $n$ such that $M_{S}^{2}\left(N_{S}\right), M_{P}^{2}\left(N_{P}\right) \leq \Lambda_{c o}^{2}$, while $M_{S}^{2}\left(N_{S}+1\right), M_{P}^{2}\left(N_{P}+1\right)>\Lambda_{c o}^{2}$. Using Eq. (9), our regulated sum rule now reads

$$
16 B^{2} L_{8}\left(\Lambda_{c o}\right)=\sum_{n}^{N_{S}\left(\Lambda_{c o}\right)} f_{S}^{2}(n)-\sum_{n}^{N_{P}\left(\Lambda_{c o}\right)} f_{P}^{2}(n)
$$

This is of course finite, by construction. However, it does not satisfy the basic fieldtheoretic requirement that physics should be independent of the cutoff, i.e. that the relative change in $L_{8}$ due to a change in cutoff goes to zero as we take the cutoff to infinity. Mathematically, this requirement translates into

$$
L_{8}\left(\Lambda_{c o}\right)=L_{8}+O\left(\frac{1}{\Lambda_{c o}}\right)
$$

where $L_{8}$ is the value of the LEC in the limit of infinite cutoff. That Eq. (12) does not in general satisfy this requirement can be seen as follows. When we increase $\Lambda_{c o}$, both $N_{S}$ and $N_{P}$ increase by integer steps when $\Lambda_{c o}$ moves past the next resonance mass in either channel. Suppose that we increase $\Lambda_{c o}$ such that $N_{S}$ changes by 1 to $N_{S}^{\prime}=N_{S}+1$, while $N_{P}^{\prime}=N_{P}$ stays the same. This leads to a change $\Delta L_{8}$ in $L_{8}$ equal to

$$
\Delta L_{8}=\frac{f_{S}^{2}\left(n=N_{S}+1\right)}{16 B^{2}}
$$

We do not at present have enough information about QCD allowing us to conclude that the right-hand side of this equation goes to zero for $\Lambda_{c o} \rightarrow \infty$. In fact, it is not the case for the Regge-like behavior of Eq. (7), for which $f_{S}^{2}(n)$ goes to a constant for large $n\left(c f\right.$. Eq. (11)). Therefore, we conclude that the sum rule for $L_{8}$ as given by Eq. (6) , even with the cutoff we introduced to regulate the sums in Eq. (6), is not well-defined. ${ }^{3}$

\subsection{A better sum rule for $L_{8}$}

Obviously, the invalid step in the derivation of the sum rule (6) is the interchange of the integral over $t$ and the sum over $n$, as this led to an expression containing the difference of two quadratically divergent sums. We may remedy this problem by starting from a once-subtracted dispersion relation for $\Delta \Pi\left(q^{2}\right)$ [6],

$$
\Delta \Pi\left(q^{2}=-Q^{2}\right)=\Delta \Pi(0)-\frac{Q^{2}}{\pi} \int_{0}^{\infty} d t \frac{\frac{1}{2} \operatorname{Im}\left(\Pi_{S}(t)-\Pi_{P}^{\prime}(t)\right)}{t\left(t+Q^{2}\right)},
$$

where again the pion pole has been omitted. Substituting the large- $N_{c}$ expressions Eq. (4) for the spectral functions, one obtains

$$
\Delta \Pi\left(q^{2}=-Q^{2}\right)=\Delta \Pi(0)-Q^{2}\left(\sum_{n}^{N_{S}} \frac{f_{S}^{2}(n)}{M_{S}^{2}(n)+Q^{2}}-\sum_{n}^{N_{P}} \frac{f_{P}^{2}(n)}{M_{P}^{2}(n)+Q^{2}}\right),
$$

\footnotetext{
${ }^{3}$ Unless there is an $N$ so that $f_{S}(n)=f_{P}(n)$ for all $n>N$.
} 
where we used Eq. (9), and where $N_{S}$ and $N_{P}$ are to be taken to infinity. Again we interchanged the integral over $t$ with the sum over $n$, but now each sum in Eq. (16) is only logarithmically divergent:

$$
\sum_{n}^{N} \frac{f^{2}(n)}{M^{2}(n)+Q^{2}} \sim \int^{M^{2}(N)} d M^{2}\left(\frac{d n}{d M^{2}} f^{2}(n)\right) \frac{1}{M^{2}+Q^{2}} \sim \kappa \log \left(M^{2}(N) / Q^{2}\right)
$$

(cf. Eq. (11)), and therefore a cutoff $\Lambda_{c o}$ needs to be introduced such that $Q^{2} \ll \Lambda_{c o}^{2} \sim$ $M_{S}^{2}\left(N_{S}\right) \sim M_{P}^{2}\left(N_{P}\right)$. The limit of $N_{S}$ and $N_{P}$ to infinity thus has to be taken in a correlated way such that this condition is fulfilled. It follows that the difference in Eq. (16) is finite and unambiguous. Using the fact that $\Delta \Pi\left(Q^{2}\right)$ should vanish for asymptotically large $Q^{2}$, we arrive at a fully regulated sum rule for $L_{8}$, valid in large- $N_{c}$ QCD: ${ }^{4}$

$$
16 B^{2} L_{8}=\Delta \Pi(0)=\lim _{Q^{2} \rightarrow \infty} Q^{2} \lim _{N_{s}, N_{P} \rightarrow \infty}\left(\sum_{n}^{N_{S}} \frac{f_{S}^{2}(n)}{M_{S}^{2}(n)+Q^{2}}-\sum_{n}^{N_{P}} \frac{f_{P}^{2}(n)}{M_{P}^{2}(n)+Q^{2}}\right),
$$

where it is understood that the limit $N_{S}, N_{P} \rightarrow \infty$ is taken in a correlated way, such that $M_{S}^{2}\left(N_{S}\right) / M_{P}^{2}\left(N_{P}\right) \rightarrow 1$.

If, as already discussed in Sec. 2.1, we take the number of resonances in each channel to be finite, assuming that there are $n_{S}$ in the scalar channel and $n_{P}$ in the pseudoscalar channel below a certain scale $s_{0}$, above which we stipulate that the scalar and pseudoscalar two-point spectral functions exactly cancel, each of the sums in Eq. (18) is finite, and we may take the large- $Q^{2}$ limit under the sums, obtaining

$$
16 B^{2} L_{8}=\sum_{n=1}^{n_{S}} f_{S}^{2}(n)-\sum_{n=1}^{n_{P}} f_{P}^{2}(n)
$$

i.e. a finite version of Eq. (6). In general, if the difference of the sums in Eq. (18) would be sufficiently convergent, one would be allowed to take the large- $Q^{2}$ limit under the sums. However, it is known that the coefficients of the OPE in QCD have anomalous dimensions, implying that logarithmic corrections to the inverse powers of $Q^{2}$ appear. This already implies that expanding in $1 / Q^{2}$ under the sums is in general not allowed. In particular, an exact cancellation of the spectral functions above some $s_{0}$ cannot occur.

It is instructive to compare our sum rule for $L_{8}$ with a similar sum rule for $L_{10}$, which is related to the difference of the vector and axial-vector two-point functions $\Pi_{V}\left(q^{2}\right)$ and $\Pi_{A}\left(q^{2}\right)$ at $q^{2}=0$. The key difference is that in this case each of these two-point functions is only logarithmically divergent to begin with, because of gauge invariance. The vector and axial-vector two-point functions are defined by

$$
\Pi_{V, A}^{\mu \nu}=i \int d x e^{i q x}\left\langle 0\left|T\left\{J_{V, A}^{\mu}(x) J_{V, A}^{\dagger \nu}(0)\right\}\right| 0\right\rangle=\left(q_{\mu} q_{\nu}-g_{\mu \nu} q^{2}\right) \Pi_{V, A}\left(q^{2}\right)
$$

\footnotetext{
${ }^{4}$ The pion pole does not play any role in this sum rule.
} 
with $J_{V}^{\mu}(x)=\bar{d}(x) \gamma_{\mu} u(x)$ and $J_{A}^{\mu}(x)=\bar{d}(x) \gamma_{\mu} \gamma_{5} u(x)$. Perturbation theory tells us that these two-point functions should both behave like $\kappa^{\prime} \log Q^{2}$ for asymptotically large $Q^{2}$, with $\kappa^{\prime}$ another constant fixed by the parton model logarithm for this case. For large $N_{c}$, the spectra look like

$$
\begin{aligned}
& \frac{1}{\pi} \operatorname{Im} \Pi_{V}(t)=2 \sum_{n}^{\infty} F_{V}^{2}(n) \delta\left(t-M_{V}^{2}(n)\right), \\
& \frac{1}{\pi} \operatorname{Im} \Pi_{A}^{\prime}(t)=2 \sum_{n}^{\infty} F_{A}^{2}(n) \delta\left(t-M_{A}^{2}(n)\right),
\end{aligned}
$$

where again the prime indicates that we omit the pion pole. A line of reasoning similar to that of Sec. 2.1 shows that

$$
\kappa^{\prime} \equiv \lim _{n \rightarrow \infty} \frac{d n}{d M_{V}^{2}} F_{V}^{2}(n)=\lim _{n \rightarrow \infty} \frac{d n}{d M_{A}^{2}} F_{A}^{2}(n) .
$$

From this it follows that both $\Pi_{V}$ and $\Pi_{A}^{\prime}$ are logarithmically divergent, so that no subtraction analogous to that in Eq. (15) is needed. We thus obtain the sum rule

$$
\begin{aligned}
-4 L_{10} & =\frac{1}{2}\left(\Pi_{V}(0)-\Pi_{A}^{\prime}(0)\right) \\
& =\lim _{N_{V}, N_{A} \rightarrow \infty}\left(\sum_{n}^{N_{V}} \frac{F_{V}^{2}(n)}{M_{V}^{2}(n)}-\sum_{n}^{N_{A}} \frac{F_{A}^{2}(n)}{M_{A}^{2}(n)}\right),
\end{aligned}
$$

where the limit $N_{V}, N_{A} \rightarrow \infty$ is again taken in a correlated way such that $\Lambda_{c o}^{2} \sim$ $M_{V}^{2}\left(N_{V}\right) \sim M_{A}^{2}\left(N_{A}\right)$ [7, 8]. Now, if we increase $\Lambda_{c o}$ such that, say, $N_{V} \rightarrow N_{V}^{\prime}=N_{V}+1$ while $N_{A} \rightarrow N_{A}^{\prime}=N_{A}, L_{10}$ changes by $\Delta L_{10}=-F_{V}^{2}\left(n=N_{V}+1\right) / 4 M_{V}^{2}\left(n=N_{V}+1\right)$. If we assume that $M_{V}^{2}(n)$ grows like $n$ (as in Regge-like behavior), we have that $\Delta L_{10} \sim$ $1 / N_{V} \rightarrow 0$ for $N_{V} \rightarrow \infty$. This is to be compared with the case of $L_{8}$ in Eq. (14).

\section{$3 \quad$ A Regge-like model at finite $N_{c}$}

Clearly, the infinite sums in Eq. (41) are not well-defined mathematical expressions. However, at finite $N_{c}$ the Dirac delta distributions should become better behaved, and it is therefore illustrative to consider the case of finite $N_{c}$. In order to do this, we will consider a simple Regge-like model. In this model, each scalar resonance will be represented by a pole of the form [9, 10]

$$
\frac{2 F_{S}^{2}(n)}{z \Lambda^{2}+M_{S}^{2}(n)},
$$

with

$$
z=\left(\frac{-q^{2}-i \epsilon}{\Lambda^{2}}\right)^{\zeta}, \quad \zeta=1-\frac{a}{\pi N_{c}}
$$


and similar for the pseudoscalar resonances, with masses and residues given by

$$
\begin{aligned}
M_{S, P}^{2}(n) & =m_{S, P}^{2}+n \Lambda^{2}, \quad n=0,1, \ldots, \\
F_{S, P}^{2}(n) & =\kappa \Lambda^{2} M_{S, P}^{2}(n),
\end{aligned}
$$

with $m_{S, P}$ also of order $1 \mathrm{GeV}(c f$. Eq. (17)). For simplicity, we take the parameters $\Lambda$ and $a$ the same in both channels. This choice satisfies Eq. (11).

Expanding the denominator of Eq. (24) to leading order in $1 / N_{c}$, there are poles near $q^{2}=M_{S, P}^{2}(n)\left(1-i a / N_{c}\right)$. Our model thus describes resonances with masses $\sim M_{S, P}(n)$ and decay widths

$$
\Gamma_{S, P}(n) \sim a M_{S, P}(n) / N_{c}
$$

In the limit $N_{c} \rightarrow \infty$, all resonances are stable. The function (24) has the correct analytic behavior in the complex $q^{2}$ plane [9]: it is analytic everywhere on the physical sheet, except for a cut along the positive real axis, starting at $q^{2}=0[9$. This model does not include all corrections one expects at finite $N_{c}$; in particular, it does not include the multi-particle continuum starting at $q^{2}=0$ due to coupling of the sources $J_{S, P}$ to pions, which would make $L_{8}$ run. However, as we will see below, the included finite- $N_{c}$ behavior already leads to an interesting observation.

The scalar spectral function for our model is given by (for simplicity, we set the scale $\Lambda$ equal to one in the rest of this section $)^{5}$

$$
\frac{1}{\pi} \operatorname{Im} \Pi_{S}(t)=\frac{1}{\pi} \sum_{n=0}^{\infty} \frac{2 F_{S}^{2}(n)\left(t^{\zeta} \sin (\zeta \pi)+\epsilon\right)}{\left(t^{\zeta} \cos (\zeta \pi)+M_{S}^{2}(n)\right)^{2}+\left(t^{\zeta} \sin (\zeta \pi)+\epsilon\right)^{2}},
$$

which for $N_{c} \rightarrow \infty$, i.e. $\zeta \rightarrow 1$, reproduces Eq. (44). The expression for $\operatorname{Im} \Pi_{P}^{\prime}(t)$ is similar. These spectral functions are logarithmically divergent with $n$, and we thus introduce a cutoff $N \equiv N_{S}=N_{P}+c$ with $c$ a finite arbitrary constant, so that $M_{S}^{2}\left(N_{S}\right) / M_{P}^{2}\left(N_{P}\right) \rightarrow 1$ for $N \rightarrow \infty .^{6}$ The difference between the scalar and pseudoscalar spectral functions is well defined, and we have that, starting from the dispersion relation in Eq. (3),

$$
\Delta \Pi(0)=\frac{\kappa}{\pi} \int_{0}^{\infty} \frac{d t}{t} \sum_{n=0}^{\infty}\left(\frac{\left(n+m_{S}^{2}\right) t^{\zeta} \sin (\zeta \pi)}{\left(t^{\zeta} \cos (\zeta \pi)+n+m_{S}^{2}\right)^{2}+\left(t^{\zeta} \sin (\zeta \pi)\right)^{2}}-(S \rightarrow P)\right)
$$

where we set $\epsilon=0$, which we are allowed to do as long as we keep $N_{c}$ finite. We may also send $N_{c}$ to infinity, but in that case we need to keep an infinitesimal $\epsilon$ in order to reproduce the Dirac $\delta$-functions of Eq. (44).

\footnotetext{
${ }^{5}$ Note that we start our sums at $n=0$, whereas in Sec. 3 of Ref. [6] sums start at $n=1$.

${ }^{6}$ We may choose $N_{S}=N_{P}+c$ with $c$ an arbitrary finite constant because nothing will depend on $c$ in the limit of infinite cutoff [8]
} 
Substituting $y=t^{\zeta}$ and performing the sum over $n$, we obtain

$$
\begin{aligned}
16 B^{2} L_{8} & =\Delta \Pi(0) \\
& =\frac{\kappa}{2 i \pi \zeta} \int_{0}^{\infty} d y\left(e^{-i \pi \zeta} \psi\left(y e^{-i \pi \zeta}+m_{S}^{2}\right)-e^{i \pi \zeta} \psi\left(y e^{i \pi \zeta}+m_{S}^{2}\right)-(S \rightarrow P)\right) \\
& =\frac{\kappa}{2 i \pi \zeta}\left[\log \Gamma\left(y e^{-i \pi \zeta}+m_{S}^{2}\right)-\log \Gamma\left(y e^{i \pi \zeta}+m_{S}^{2}\right)-(S \rightarrow P)\right]_{0}^{\infty} \\
& =\kappa\left(m_{P}^{2}-m_{S}^{2}\right),
\end{aligned}
$$

where

$$
\psi(z)=\frac{d}{d z} \log \Gamma(z)
$$

is the digamma function. ${ }^{7}$ Interestingly, in this simple model, the result is independent of $N_{c}$, and it is thus also valid for $N_{c} \rightarrow \infty{ }^{8}$ The technical reason for this is that setting $Q^{2}=0$ in order to obtain $L_{8}$ removes the dependence on $N_{c}$, which only appears through the exponent $\zeta$. We note that the new sum rule Eq. (18) does apply to our model at finite $N_{c}$ as well, if one simply replaces $Q^{2}$ by $z \Lambda^{2}$ with $z$ as defined in Eq. (25), and then takes the limit $z \rightarrow \infty$ in Eq. (18).

Let us comment on this result. First, if we calculate $L_{8}$ for this simple model using our new sum rule, Eq. (18), we obtain exactly the same result. ${ }^{9}$ Second, a key point in the calculation of Eq. (30) is that we first performed the sum over $n$, and after that the integral over $t$. These operations do not commute: if we would interchange the sum and the integral, the result would be different. If we substitute $y=t^{\zeta}$ in Eq. (29) and integrate term by term over $y$, we obtain for each term

$$
\frac{\kappa}{\pi \zeta} \int_{0}^{\infty} d y \frac{\left(n+m_{S}^{2}\right) \sin (\pi \zeta)}{\left(y \cos (\pi \zeta)+n+m_{S}^{2}\right)^{2}+(y \sin (\pi \zeta))^{2}}=\kappa,
$$

and, restoring $\Lambda$, one would thus find that

$$
\Pi_{S-P}^{\prime}(0)=\kappa \Lambda^{2}\left(\sum_{n=0}^{N} 1-\sum_{n=0}^{N-c} 1\right)=c \kappa \Lambda^{2} .
$$

Clearly, the result (33) does not equal the correct result, Eq. (30). Moreover, it depends on the undetermined constant $c$, i.e. on the details of the regulator. If, as in Sec. 2.1, we increase $\Lambda_{c o}, c$ alternates between the values 0 and 1 (taking $m_{S}^{2}<m_{P}^{2}$ ), and the value of $16 B^{2} \Delta L_{8}$ defined in Eq. (14) between $\kappa \Lambda^{2}$ and 0 . This simple example calculation demonstrates precisely what goes wrong in the derivation of the naive sum rule Eq. (6) : as we already noted in Sec. 2.1, that equation is obtained by first performing the $t$ integrals in Eq. (5) (without subtraction), and then the sums over $n$, thus leading to the difference of two quadratically divergent sums. The derivation of the new sum rule (18) avoids this problem by starting from a subtracted dispersion relation, Eq. (15).

\footnotetext{
${ }^{7}$ Our explicit calculation refutes the claim of Ref. [11] that $L_{8}$ vanishes in this type of model.

${ }^{8}$ We would obtain the same result setting $N_{c}=\infty$ from the outset, but taking $\epsilon \rightarrow 0$ at the end of the calculation.

${ }^{9}$ This calculation was done in Sec. 4 of Ref. [6].
} 


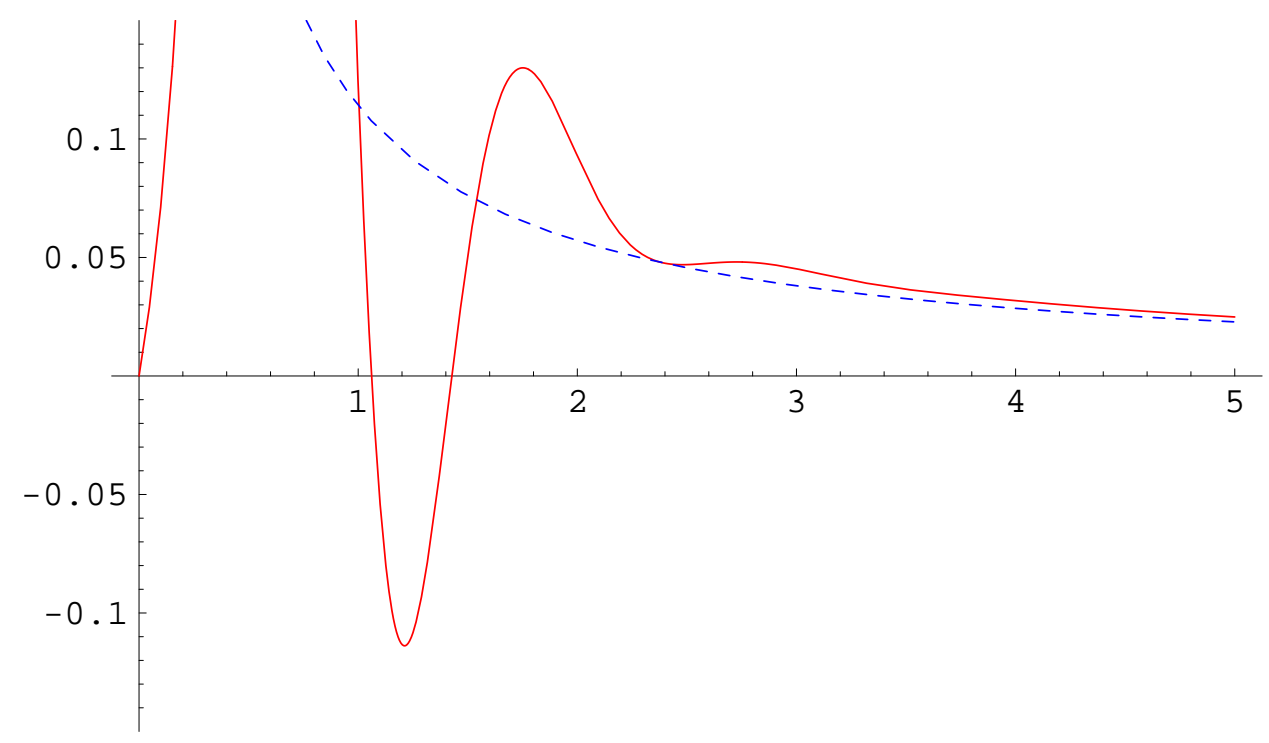

Figure 1: Plot of $y$ times the function in Eq. (30) with $m_{S}^{2}=(0.8167)^{2}, m_{P}^{2}=(1.083)^{2}$ and $\zeta=0.82$, as a function of $y$ in units in which $\Lambda=1$, with arbitrary units on the vertical axis (solid line). The dashed line is the OPE to leading order.

Finally, we observe that in our model the result is independent of $N_{c}$, and thus it is equally valid for infinite as well as finite $N_{c}$. In this respect, it is interesting to consider the spectral function at finite $N_{c}$, which we plotted in Fig. 1. More precisely, Fig. 1 is a graph of $y$ times the integrand of Eq. (30) as a function of $y$, for a value of $\zeta$ close to unity. We see that the asymptotic behavior predicted by the OPE sets in already before $y=3$, and that the resonances at higher values are completely "washed out" by their growing decay widths. In contrast, at infinite $N_{c}$ the same plot would show an infinite sequence of Dirac delta functions, cf. Eq. (4). Nevertheless, despite this very big difference in the behavior of the spectral functions at finite and infinite $N_{c}$, the value of $L_{8}$ is the same in both cases. Even though the perturbative tail cancels out in the difference between the scalar and pseudoscalar channels, $L_{8}$ is not saturated by a finite number of resonances in this model. The contribution to the integral in Eq. (30) coming from the region where the asymptotic behavior already sets in is equally important as that from the region where resonances are clearly visible.

Our example model can be made more realistic by including a finite number of resonances in the scalar and pseudoscalar channels below a certain scale $s_{0}$ which are not part of the equally-spaced towers of Eq. (24) (so that $m_{S, P}^{2}>s_{0}$ ). In that case, with $n_{S(P)}$ extra states in the scalar (pseudoscalar) channel, we would obtain

$$
16 B^{2} L_{8}=\sum_{n=1}^{n_{S}} f_{S}^{2}(n)-\sum_{n=1}^{n_{P}} f_{P}^{2}(n)+\kappa\left(m_{P}^{2}-m_{S}^{2}\right) .
$$

This is to be compared to Eq. (19). The extra term in Eq. (34) originates (in our model) from the infinite towers present above $s_{0}$. While we do not know what this extra term will look like in the case of QCD, we do know that at large $N_{c}$ there is an 
infinite number of resonances in each channel. Therefore, the omission of a term like this in Eq. (19) would represent an unknown systematic error in the application of this sum rule to QCD.

While we certainly do not expect our model to be very realistic, it turns out to be interesting to apply it to a numerical example, already in the large- $N_{c}$ limit. In order to do this, we first want to eliminate the unknown ratio $\kappa / B^{2}$ from Eq. (30). Requiring absence of a $1 / Q^{2}$ term in the OPE expression for $\Delta \Pi\left(Q^{2}\right)$ in this model, one obtains the additional relation []$^{10}$

$$
2 B^{2} f^{2}=\kappa\left(m_{S}^{2}+m_{P}^{2}-\Lambda^{2}\right)\left(m_{P}^{2}-m_{S}^{2}\right),
$$

where $f=f_{\pi}$ in the chiral limit. Combining this with Eq. (30), we find

$$
L_{8}=\frac{1}{8} \frac{f^{2}}{m_{S}^{2}+m_{P}^{2}-\Lambda^{2}} .
$$

Taking $m_{S}=0.98 \mathrm{GeV}, m_{P}=1.3 \mathrm{GeV}, \Lambda=1.2 \mathrm{GeV}$ (determined from the $a_{0}(1450)$ and $\pi(1800)$ ) and $f=87 \mathrm{MeV}$, we find $L_{8}=8 \times 10^{-4}$ to be compared with, e.g., $L_{8}\left(M_{\rho}\right)=9 \times 10^{-4}[16]$.

Let us again contrast the case of $L_{8}$ with that of $L_{10}$, as we already did at the end of Sec. 2. In a model with an equally-spaced tower of resonances in each channel, one finds for $L_{10} 7$

$$
-4 L_{10}=\kappa^{\prime} \sum_{n=0}^{\infty} \frac{\left(m_{A}^{2}-m_{V}^{2}\right) \Lambda^{2}}{\left(m_{V}^{2}+n \Lambda^{2}\right)\left(m_{A}^{2}+n \Lambda^{2}\right)},
$$

in which $m_{V, A}$ are the lowest masses in the two towers. The key difference is that in this case the contribution from the towers is not only proportional to the difference $m_{A}^{2}-m_{V}^{2}$, analogous to the case of $L_{8}$, but also suppressed by inverse powers of the product of these resonance masses. Taking the contribution from the first few resonances in the tower may already be a good approximation. For instance, with $\kappa^{\prime}=1 / 8 \pi^{2}$ and taking $m_{V}=0.77 \mathrm{GeV}, m_{A}=1.25 \mathrm{GeV}$ and $\Lambda=1.3 \mathrm{GeV}$ gives $L_{10}=-5.6 \times 10^{-3}$, to be compared with the full sum in Eq. (37) which gives $L_{10}=-7.1 \times 10^{-3}$. The experimental estimate is $L_{10}\left(M_{\rho}\right)=-5.5 \times 10^{-3}$ [16].

\section{Saturation with a finite number of resonances}

It is often assumed that each spectral function may be approximated by a finite sum over resonances up to a certain scale $s_{0}$, and a "perturbative continuum" at values of $t>s_{0}$. The perturbative continuum then cancels between scalar and pseudoscalar channels, leaving the difference between two finite sums in Eq. (19). In these sums, the decay constants and masses of the resonances are to be considered effective parameters to be fixed by requiring consistency with the low- and high-momentum expansion of the relevant Green's function, as obtained from the chiral and operator product

\footnotetext{
${ }^{10}$ Here $m_{S, P}$ correspond to the lowest masses in the towers, whereas in Ref. 6 their values were expressed as $\sqrt{m_{S, P}^{2}+\Lambda^{2}}$.
} 
expansions, respectively [2]. In the large- $N_{c}$ limit, this phenomenological ansatz is a rational approximation [13] to QCD Green's functions, known as the Minimal Hadronic Approximation (MHA) [3]. It encompasses vector meson dominance as a particular case.

One may also attempt to use only the high-energy input from the OPE to predict the low-energy constants, which parameterize the low-momentum expansion. Although phenomenologically this assumption seems to work [16], it remains unclear how this follows from QCD. It is also important to be able to control the systematic error associated with this approximation since, in practice, it is very difficult to go beyond the inclusion of one resonance per channel. Furthermore, there are often not enough equations to determine the decay constants and the masses, so that the effective masses are actually guessed from the position of the physical masses.

In order to gain some insight into these questions a model like that in Eqs. (24 26) is useful because it allows for a comparison of the phenomenological ansatz with the exact large- $N_{c}$ results produced by the model. Therefore let us consider the ansatz

$$
\frac{1}{2}\left(\Pi_{S}\left(q^{2}\right)-\Pi_{P}\left(q^{2}\right)\right)_{M H A}=\frac{\hat{F}_{S}^{2}}{Q^{2}+\hat{M}_{S}^{2}}-\frac{\hat{F}_{P}^{2}}{Q^{2}+\hat{M}_{P}^{2}}-\frac{B^{2} f^{2}}{Q^{2}}
$$

where $\hat{M}_{S, P}$ and $\hat{F}_{S, P}$ are parameters to be determined. The idea is to use short-distance (OPE) constraints to fix these parameters, and then use their values to predict $L_{8}$ from

$$
16 B^{2} L_{8}=\frac{\hat{F}_{S}^{2}}{\hat{M}_{S}^{2}}-\frac{\hat{F}_{P}^{2}}{\hat{M}_{P}^{2}}
$$

Writing

$$
\hat{F}_{S, P}^{2}=\hat{f}_{S, P}^{2} \hat{M}_{S, P}^{2}
$$

the leading $\mathrm{OPE}\left(1 / Q^{2}\right)$ constraint is $[6]$

$$
\hat{f}_{S}^{2} \hat{M}_{S}^{2}-\hat{f}_{P}^{2} \hat{M}_{P}^{2}=\frac{\kappa}{2}\left(m_{P}^{2}-m_{S}^{2}\right)\left(m_{S}^{2}+m_{P}^{2}-\Lambda^{2}\right) .
$$

With values of $m_{S, P}$ and $\Lambda$ close enough to $1 \mathrm{GeV}$, and assuming that $m_{P}>m_{S}$, as phenomenology suggests, the right-hand side of this equation is positive. In fact, this is what happens for the values chosen after Eq. (36).

While this one equation is not sufficient to determine the MHA parameters, an interesting observation can already be made: MHA does not coincide with the physical masses and decay constants. Suppose that we look for solutions in which the decay constants take their actual values. In our model, that means $\hat{f}_{S}^{2}=\hat{f}_{P}^{2}=\kappa \Lambda^{2}$. One immediately concludes that the ordering of the mass parameters comes out reversed, i.e. $\hat{M}_{S}>\hat{M}_{P}$ while the true ordering is $m_{P}>m_{S}$. The mathematical origin of this difference in sign comes from the fact that the model contains an infinite number of resonances.

To investigate MHA in more detail, let us impose that it gives us the right value of $L_{8}$, which adds the relation

$$
\hat{f}_{S}^{2}-\hat{f}_{P}^{2}=\kappa\left(m_{P}^{2}-m_{S}^{2}\right)
$$


The lowest resonance masses are relatively well known, ${ }^{11}$ while the $f$ parameters are much less well known. If one takes the MHA masses as the physical ones, i.e. $\hat{M}_{S}=m_{S}$ and $\hat{M}_{P}=m_{P}$, one can solve for $\hat{f}_{S}$ and $\hat{f}_{P}$ to find

$$
\begin{aligned}
& \hat{f}_{S}^{2}=\frac{1}{2} \kappa\left(m_{P}^{2}-m_{S}^{2}+\Lambda^{2}\right), \\
& \hat{f}_{P}^{2}=\frac{1}{2} \kappa\left(m_{S}^{2}-m_{P}^{2}+\Lambda^{2}\right) .
\end{aligned}
$$

These solutions are very far from their "real-world" values (which, in our test, are the model values $\hat{f}_{S}^{2}=\hat{f}_{P}^{2}=\kappa \Lambda^{2}$ ). Taking the ratio, and using for instance the numerical example discussed at the end of Sec. 3, we find

$$
\frac{\hat{f}_{P}^{2}}{\hat{f}_{S}^{2}}=\frac{m_{S}^{2}-m_{P}^{2}+\Lambda^{2}}{m_{P}^{2}-m_{S}^{2}+\Lambda^{2}} \simeq \frac{1}{3}
$$

instead of 1 , which is the actual value. Note that both the low-energy constraint (42) and the high-energy constraint (411) force $\hat{f}_{S}^{2}$ to be different from $\hat{f}_{P}^{2}$ to the extent that $m_{S}$ is not equal to $m_{P}$. Remarkably, nevertheless, the MHA expression (38) approximates the true function $\Pi_{S}-\Pi_{P}$ to within a few percent for all euclidean $Q^{2}$. Similar properties of the Adler function were studied in more detail in Ref. [12].

One may also try an even simpler ansatz, in which only one scalar resonance (in addition to the pion) is kept. Solving Eqs. (41) and (42) for $\hat{f}_{S}^{2}$ and $\hat{M}_{S}^{2}$, we find, using the same numerical example, $\hat{M}_{S}^{2} \simeq 0.63 m_{S}^{2}$ and $\hat{f}_{S}^{2} \simeq 0.51 \kappa \Lambda^{2}$. As before, the MHA values are very different from the real ones, even though the rational approximation to $\Pi_{S}-\Pi_{P}$ works again within a few percent for all euclidean $Q^{2}$.

\section{Conclusion}

In this paper, we revisited the connection of the order- $p^{4}$ LEC $L_{8}$ to the scalar and pseudoscalar resonance parameters. In Sec. 2.1 we reviewed the fact that a sum rule of the form (6) is generally ill-defined, and showed that this is to be expected on general grounds in QCD. In Sec. 2.2 we derived a better sum rule, Eq. (18), and argued why this new sum rule gives a finite and universal result. Universality follows because each of the sums in Eq. (18) is only logaritmically divergent, with the difference being finite, and thus the precise details of the choice of the cutoffs $N_{P}$ relative to $N_{S}$ do not matter, as required by quantum field theory [8]. For instance, increasing $N_{S}$ to $N_{S}+1$ in Eq. (18) does not change $L_{8}$ in the limit $N_{S} \rightarrow \infty$.

Of course, the ill-defined sum rule of Eq. (6) itself is never used in practice, but instead each sum is restricted to a relatively small set of resonances below a scale $s_{0}$ with adjustable parameters, as reviewed in Sec. 4. The derivation of such sum rules, in this case Eq. (19), assumes that above a certain scale $s_{0}$ the spectral functions $\operatorname{Im} \Pi_{S}(t) / \pi$ and $\operatorname{Im} \Pi_{P}(t) / \pi$ are exactly equal. In contrast, our new sum rule, Eq. (18)

\footnotetext{
${ }^{11}$ Assuming that the lowest scalar surviving the large- $N_{c}$ limit is the $a_{0}$ with mass $0.98 \mathrm{GeV}$.
} 
relies only on the fact that $\Pi_{S}$ and $\Pi_{P}$ become equal for asymptotically large $Q^{2}$ in the euclidean regime.

In Ref. 14] it was shown that, by using the theory of Pade approximants [13], there is a mathematically well-defined connection between resonance parameters and LECs in large- $N_{c}$ QCD. However, this work is not applicable in the case of $L_{8}$ because the spectral function defining $\Delta \Pi\left(q^{2}\right)$ is not positive.

The sum rule (19) can approximate the sum rule (18) if the contribution from the resonances above a certain $s_{0}$ is very small. However, there appears to be no reason for this to be true in QCD, unless some new mechanism plays a role higher up in the spectrum. A possibility might be that the resonance parameters of $S$ and $P$ states pair up at higher scales, aligning sufficiently fast for higher resonances to almost not contribute to our sum rule, Eq. (18). This scenario of so-called "parity doubling" or "chiral symmetry restoration" has been the subject of much speculation recently, but little to nothing is known to date about its validity [15. In fact, our simple model demonstrates that this parity doubling in QCD does not follow from the cancellation of the perturbative tail in $\Pi_{S}-\Pi_{P}$, and neither does the saturation of $L_{8}$ by a finite number of resonances.

Clearly, our new sum rule is less practical if indeed the contribution from resonances above $s_{0}$ cannot be ignored. However, ignoring this contribution anyway would obviously introduce an unknown systematic error. In Sec. 3 we showed how the new sum rule works in a simple example, in which an explicit form for the contribution coming from resonances above $s_{0}$ can be derived, $c f$. Eq. (34). While Sec. 2, and in particular the sum rule of Eq. (18), apply to QCD in the limit of infinite $N_{c}$, our model is defined for any value of $N_{c}$. It is semi-realistic in the sense that it has consistent analytic behavior in the whole complex $q^{2}$ plane, while it predicts widths which are suppressed like $1 / N_{c}$, but which grow with increasing resonance mass. In particular, we point to Fig. 1, which shows a qualitatively realistic spectral function for the two-point function $\Delta \Pi\left(q^{2}\right)$. Interestingly, the result for $L_{8}$ in that model, Eq. (30), is independent of $N_{c}$. We thus speculate that also at finite $N_{c}$ our general conclusion, that the region above $s_{0}$ cannot be ignored, remains valid.

Phenomenologically, Eq. (39) seems to work quite well [16]. As we have argued in Sec. 4 using our Regge-like model, this may be at the expense of values for the parameters in the rational approximant (38), which substantially deviate from the actual ones representing the lowest resonances, but which produce a very good approximation to the function $\Pi_{S}-\Pi_{P}$ over the entire euclidean region in $Q^{2}$. Since we lack precise information on the scalar and pseudoscalar decay constants in the real world, such deviations might go unnoticed. We conclude that, in spite of the fact that the phenomenology of the scalar/pseudoscalar sector looks very reasonable, a deeper understanding of the relation between $L_{8}$ and the spectrum in the scalar and pseudoscalar sectors in large- $N_{c}$ QCD remains an interesting puzzle. 


\section{Acknowledgements}

We thank Oscar Catà, Matthias Jamin, Toni Pich and Eduardo de Rafael for useful discussions. SP is supported in part by CICYT-FEDER-FPA2005-02211 and SGR200500916, and MG is supported in part by the Generalitat de Catalunya under the program PIV1-2005 and by the US Department of Energy.

\section{References}

[1] J. Gasser and H. Leutwyler, Nucl. Phys. B 250, 465 (1985).

[2] G. Ecker, J. Gasser, A. Pich and E. de Rafael, Nucl. Phys. B 321, 311 (1989); G. Ecker, J. Gasser, H. Leutwyler, A. Pich and E. de Rafael, Phys. Lett. B 223, 425 (1989);

[3] M. Knecht and E. de Rafael, Phys. Lett. B 424, 335 (1998) arXiv:hep-ph/9712457; S. Peris, M. Perrottet and E. de Rafael, JHEP 9805, 011 (1998) arXiv:hep-ph/9805442 . See also S. Peris, arXiv:hep-ph/0204181 and E. de Rafael, Nucl. Phys. Proc. Suppl. 119, 71 (2003) arXiv:hep-ph/0210317|.

[4] See for instance J. Bijnens, E. de Rafael and H. Zheng, Z. Phys. C 62, 437 (1994) arXiv:hep-ph/9306323.

[5] G. 't Hooft, Nucl. Phys. B 72, 461 (1974); E. Witten, Nucl. Phys. B 160, 57 (1979).

[6] O. Catà, M. Golterman and S. Peris, Phys. Rev. D 74, 016001 (2006) arXiv:hep-ph/0602194.

[7] M. Golterman and S. Peris, JHEP 0101, 028 (2001) arXiv:hep-ph/0101098.

[8] M. Golterman and S. Peris, Phys. Rev. D 67, 096001 (2003) arXiv:hep-ph/0207060.

[9] B. Blok, M. A. Shifman and D. X. Zhang, Phys. Rev. D 57, 2691 (1998) [Erratum-ibid. D 59, 019901 (1999)] arXiv:hep-ph/9709333; I. I. Y. Bigi, M. A. Shifman, N. Uraltsev and A. I. Vainshtein, Phys. Rev. D 59, 054011 (1999) arXiv:hep-ph/9805241.

[10] O. Catà, M. Golterman and S. Peris, JHEP 0508, 076 (2005) arXiv:hep-ph/0506004.

[11] S. S. Afonin and D. Espriu, arXiv:hep-ph/0602219.

[12] M. Golterman, S. Peris, B. Phily and E. De Rafael, JHEP 0201, 024 (2002) arXiv:hep-ph/0112042.

[13] For the mathematical theory of rational approximants see, e.g., G.A. Baker and P. Graves-Morris, "Pade Approximants," Cambridge Univ. Press 1996. 
[14] S. Peris, arXiv:hep-ph/0603190.

[15] See R. L. Jaffe, D. Pirjol and A. Scardicchio, arXiv:hep-ph/0602010, and references therein.

[16] A. Pich, Int. J. Mod. Phys. A 20, 1613 (2005) arXiv:hep-ph/0410322. 